\title{
Specification and Top Down Design \\ of \\ Distributed systems
}

\author{
Manfred Broy \\ Fakultat for Mathematik und Informatik \\ Universitst Passau \\ Postfach 2540 \\ 8390 Passau
}

\begin{abstract}
Stream-processing functions provide an excellent semantic model for the abstract representation of systems of nondeterministic concurrent communicating agents. Based on this model a formalism for the specification of such functions is suggested. This way a fully modular, compositional methodology for the specification and the design of distributed systems and their components is derived. Concepts of correctness are defined and rules of inference are discussed that help to transform such specifications into a network of communicating agents. A combinatorial ("functional") notation for the sequential and parallel composition as well as feedback for those agents is introduced.
\end{abstract}

\section{Introduction}

For the top-down design of concurrent communicating systems a spectfication formalism is an indispensible requisite. Only if one is able to give modular specifications, i.e. abstract specifications also for the subcomponents of a distributed system, then the decomposition of the system can be done properly and the subcomponents can be developed and verified separately.

In this paper we are not interested in the internal structure of (components of) concurrent communicating systems ("internal behaviour") that could be described for instance by event structures ( $\mathrm{cf}$. (Winskel 82]) but rather in the "input/output" behaviour of these systems ("extensional behaviour").

In the following a simple language formalism is suggested that can be used as a formal framework for the specification, design and verification of concurrent, communicating systems and their components. It is based on a semantic model for concurrent, communicating systems and is an attempt to combine ideas and concepts from denotational semantics for concurrent systems (cf. [Broy 83b]), programming logic (such as temporal logic, of. [Pnueli 77]), algebraic specifications (of. [Broy, Wirsing 82]), program transformation (cf. [Broy 84d]), and functional (multi-) programming (of. [Broy 81]). 


\section{A Specification Formalism}

In this section a specification formalism is introduced that allows to specify communicating agents with a finite set of input lines and a finite set of output lines. We start by giving some examples, then give a formal syntax and finally define the semantics.

\subsection{Specifications of Comaunicating Agents}

A communicating agent has $n$ input lines and $m$ output lines, where $m$ and $n$ are arbitrary natural numbers (including 0 ). On every input line a finite or infinite sequence of data is transmitted to the agent and on every output line a finite or infinite sequence of data is generated by the agent. The input lines and output lines have internal (local) names that are used in a predicate for expressing the relationship between the input and output.

\subsubsection{First Examples for Specifications of Agents}

We start with the example of a very simple agent store which nevertheless shows the full power of the method:

agent store $=$ input stream data $d$, stream bool b, output stream data $r$,

first $b \Rightarrow r=$ store (rest $d$, rest $b$ ),

$\neg$ first $b \Rightarrow r=$ first $d \& \operatorname{store}(d$, rest $b)$ end

This specification defines an agent with 2 input lines and 1 output line:



The identifiers $d, b, r$ for the input lines and output lines are only internal ("bound") names and not relevant to the outside. The operator \& puts an element in front of a sequence, first $s$ returns the first element of a sequence $s$, rest $s$ returns the sequence $s$ without the first element.

According to the specification taking the sequences

$d=1 \& 2 \& 3 \& 4 \& \ldots$

$b=$ false \& false \& true \& false \& true \& false \& ...

as input implies the output sequence

$$
r=1 \& 1 \& 2 \& 3 \& \ldots
$$

The agent store may be seen as a memory cell where $b$ can be interpreted as the read/write command sequence: If the input on input line $b$ is true, then a new data value on the input line $d$ is stored, if the input on input line $b$ is $f a l s e$, then this is interpreted as a read command: the current input on $d$ is copied as output. 
As a second example the agent schedule is specified:

agent schedule $=$

input stream data $a$, stream data $b$, stream bool $s$, output stream data $r$,

first $s \Rightarrow r=$ first a \& schedule (rest $a, b$, rest $s$ ),

$\neg$ first $s \Rightarrow r=$ first $b \&$ schedule $(a$, rest $b$, rest $s$ ) end

The agent schedule receives three input streams: two data streams and one stream of booleans. The data streams are merged according to the boolean values in the third input stream. With the input

$a=0 \& 2 \& 4 \& \ldots$

$\mathrm{b}=1 \& 3 \& 5 \& \ldots$

$s=$ true \& true \& false \& false \& true \& false \& ...

the agent schedule produces

$r=0 \& 2 \& 1 \& 3 \& 4 \& 5 \& \ldots$

Another example is the agent switch:

agent switch $=$ input stream data $s$, stream bool $b$, output stream data $r 1, r 2$,

$\forall$ stream data $d 1, d 2:(d 1, d 2)=\operatorname{switch}($ rest $s$, rest $b) \Rightarrow$ (first $b \Rightarrow(r 1=$ first $s \& d 1 \wedge d 2=r 2)) \wedge$ $\left(\neg\right.$ first $b \Rightarrow\left(d 1=r 1 \wedge r^{2}=\right.$ first $\left.\left.s \& d 2\right)\right) \quad$ end

The agent switch produces two output streams by sending the input on its input line $s$ either to the left or to the right output line depending on the boolean input in its input line b. One may prove

$\operatorname{switch}(s, b)=(d 1, d 2) \Rightarrow \operatorname{schedule}(d 1, d 2, b)=s$.

An agent even may have no input lines at all. An example reads:

agent onestream $=$ output stream nat $r, r=1 \& r$

end

The agent onestream produces an infinite stream of 1 's:

$r=1 \& 1 \& 1 \& \ldots$

One may write also agents that perform arithmetic operations on its input streams. A simple example is:

agent addstream $=$ input stream nat $a$, strean nat $b$, output stream nat $r$,

$$
r=(\text { first a +first b) \& addstream (rest a, rest b) end }
$$

This agent adds the sequences of input streams elementwise; if

$a=0 \& 2 \& 4 \& \ldots$

$b=1 \& 3 \& 5 \& \ldots$ 
then one obtains

$r=1 \& 5 \& 9 \& \ldots$

Agents may also be specified based on other agents

agent natstream =

output strean nat $r, r=0$ \& addstream( $r$, onestream) end

The agent natstream produces the infinite stream of the natural numbers:

$r=0 \& 1 \& 2 \& \ldots$

So far the specified agents looked more or less deterministic, i.e. uniquely specified. They precisely describe functions from the tuple of input streams to the tuple of output streams.

\subsubsection{Nondeterministic Agents}

of course, nondeterministic agent specifications may be written in the formalism, too.

agent infinite $=$ output strean bool $r, r=$ true \& $r \quad r \quad r=$ false \& $r$ end

The agent infinite has two possible output streams:

$r=$ true \& true \& $\ldots$ or $r=$ false \& false \& $\ldots$

The possibility of writing nondeterministic agent specifications leads to the possibility (and the problems) of using nondeterministic expressions. For instance with the specification above the agent infinite produces nondeterministically one of the two infinite sequences. This leads to the problem of nondeterministic terms and their meaning. Using nondeterministic terms in equations brings a number of complications. Writing

$t 1=t 2$

for nondeterministic terms $t 1$ and $t 2$ what does that mean? That $t .1$ and t.2 must stand for the same set of possible values? For showing some of the subtle differences consider:

agent any $=$ output strean bool $r, r=$ true \& any() $\vee r=$ false \& any() end

The agent any produces any infinite sequence of boolean values. Another even more famous and more important example is the agent merge:

agent merge = input stream data $a$, stream data $b$, output stream $r$

$\exists$ stream bool $s: s=\operatorname{any}() \wedge r=\operatorname{schedule}(a, b, s)$ end The agent merge is highly nondeterministic. For instance with the infinite streams

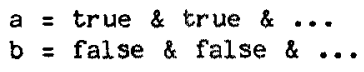


the term merge( $a, b)$ may stand for any sequence of boolean values.

We take here a very liberal viewpoint for equations between nondeterministic terms, i.e. terms in which nondeterministic agents occur. With every agent a set of stream processing functions is associated. An equation between terms using agents is fulfilled, if for every occurrence of an agent identifier one may find a stream-processing function in the given set associated with the agent identifier, such that the equations are fulfilled.

After having given a number of simple examples the syntax and the semantics of the specification language is now formally defined.

\subsection{Syntactic Fora of an Agent Specification}

In this section a syntax for spęcifications of communicating agents is given in BNF-style notation.

\section{Syntax}



In this syntax it is assumed

- a set 〈id〉 of identiflers for data or streams of data,

- a set 〈agent-id> of identifiers for agents,

- a set 〈primitive functions〉 of identifiers for primitive (given) agents.

of course a number of context conditions (such as well-formedness or type-correctness of terms) have to be presupposed for ensuring that an agent specification is meaningful. For convenience and lack of space context conditions are not given explicitly. 


\subsection{Semantics of Agent Specifications}

In this section a semantic model is introduced, based on an algebra of primitive data and functions. The semantic model consists essentially of stream processing functions. Then the agent specification formalism is related to this semantic model.

\subsubsection{Streams and Stream-processing Functions}

As one of the most fundamental domains for communicating programs one may consider the domain of streams (cf. [Broy 81]). Given a flat domain A 1 (i,e. a set $A$, with $\perp A, A^{\top}=A \cup\{1\}$, ordered by a 1 a2 iff a $1=a 2 \vee$ a $1=1$ for $a 1, a 2 \in A+$ ) the domain $\operatorname{STREAM}(A)$ of streams over $A$ is defined by

$$
\operatorname{STREAM}(A)=\left(A^{*} \times(1\}\right) \cup A^{*} \cup A^{\infty}
$$

where $A^{*}$ denotes the finite sequences (words) over $A$, and $A \infty$ denotes the infinite sequences (words) over A. For 51,32 a STREAM(A) a partial ordering is defined by

$$
s 1 \text { [ s2 iff } s 1=s 2 \text { or } 3 s 3 \in A^{*}, \begin{array}{r}
s 4 \in \operatorname{STREAM}(A): \\
s 1=53^{\circ}\langle 1\rangle \wedge
\end{array}
$$

Here "On denotes the usual concatenation where for $s \in A \infty$ we define $s^{0} s^{\prime}=s$; for all a $c A$ by $\langle a\rangle$ we denote the one-element sequence consisting of $a$. By 8 we denote the empty sequence.

With these definitions (STREAM(A), [) forms an algebraic domain where $\left(A^{*} \times(1\}\right)$ $\cup A^{*}$ is the set of finite elements, $A_{\infty}$ the infinite ones, $A^{*} \times\{1\}$ the partial ones and $A^{*} U A \infty$ the total ones. The stream $\langle 1\rangle$ represents the least element.

Streams can be used for representing the sequence of communications of a program, for instance the output on a specific channel. Of course for nonterminating programs the output may be an infinite sequence.

For comranicating programs one has to distinguish two forms of nontermination: nontermination with infinite output and nontermination without any further output. The first is represented by an infinite stream, the second by a finite stream ending with the 1 -symbol. In this case one may speak of divergence.

The following four basic functions are used on streams:

$$
\begin{aligned}
& \text { ap } \quad \text { : } A+x \operatorname{StREAM}(A) \rightarrow \operatorname{StREAM}(A) \\
& \text { rest : } \operatorname{STREAM}(A) \rightarrow \operatorname{STREAM}(A) \\
& \text { first : } \operatorname{STREAM}(A) \rightarrow A^{4} \\
& \text { isempty } \quad: \operatorname{STREAM}(A) \rightarrow \mathbb{B}+
\end{aligned}
$$

defined by

$$
a p(a, s)= \begin{cases}\langle a\rangle \circ s & \text { if } a<A, s a \operatorname{STREAM}(A) \\ \langle 1\rangle & \text { otherwise }\end{cases}
$$


For ap (a, s) we often write a\&s. Note that ap is a nonstrict function: the result of applying ap may be different from the least element $\langle\downarrow\rangle$ even if the second argument is $\langle\downarrow\rangle$. However, if the first argument is the least element of the domain (is $\downarrow$ ) then the result is the least element (is $\langle\downarrow\rangle$ ). The function ap is left-strict. The stream a\&s can be seen as a sequence of communicated data (for instance output). As soon as $a$ is 4 , then there cannot be any defined output afterwards: $1 \& s=\langle \&\rangle$. So the definition of ap mirrors the simple fact of communicating processes, that after divergence there cannot be any further output.

Note that STREAM(A) is not closed with respect to concatenation since for streams



Let a $A, s$ STREAM(A), then the functions rest, first, isempty are defined by:

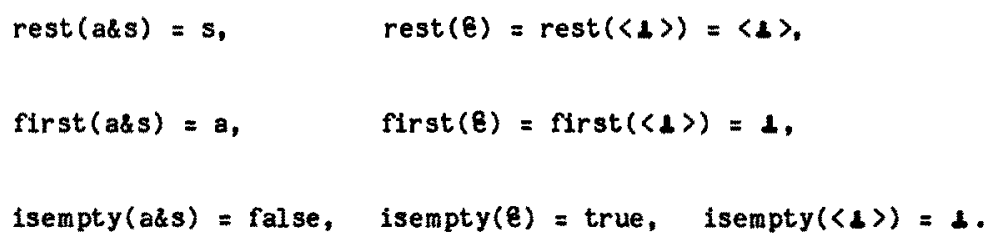

One simply proves:

Lemma: The functions ap. rest, first and isempty are monotonic and continuous.

For obvious reasons streams can be considered as one of the most fundamental domains when dealing with systems of communicating processes. For procedural concurrent programs with shared memory one may consider streams of states, for processes with explicit communication primitives one can think of streams of communication actions (cf. [Broy 83a, 84a, 84b]).

For giving meaning to agent specifications a flxed set DATA of atomic data objects is assumed. For writing examples we assume $\mathbb{N} \approx$ DATA and true, false $\leq$ DATA. In a more complete framework, one may assume some abstract data type specification method for specifying the atomic data objects.

The set $D$ of all objects on which agents operate is defined by

$D=$ def DATA 4 STREAM(DATA)

An agent with $m$ input lines and $n$ output lines is a continuous mapping. The set of those continuous mappings is defined by: 


$$
A G E N T_{n}^{M}=\left[D M \rightarrow D^{n}\right]
$$

The set of all agents is defined by

$$
\text { ACENT }=\left\{f \in\left[D^{m} \rightarrow D^{n}\right]: n, m \in \mathbb{N}\right\}
$$

Having fixed the data universe $D$ and the universe of agents $\operatorname{AGENT}_{n}^{m}$ now meaning can be assigned to specifications.

\subsubsection{Assigning Meaning to Agent Specifications}

For assigning meaning to agent specifications the well-known technique of environments is used: Two kinds of environments are needed, data environments and agent environments.

$$
\begin{aligned}
& \text { ENV }=\operatorname{def}(\langle i d\rangle \rightarrow D) \\
& \text { AENV }=\text { def }(\langle\text { agent-id }\rangle \rightarrow P(A G E T) \backslash\{\emptyset\})
\end{aligned}
$$

An agent environment associates with every agent identifier a set of agents. An environment for identifiers associates with every identifier a stream or a data object.

Note that for very particular reasons ( $c f$. concluding remarks) we have chosen to associate with every agent identifier a set of continuous stream processing functions instead of using functions mapping (tuples of) streams into sets of (tuples of) streams.

As usual the updating of environments $\sigma$ is denoted by $\sigma[d / x]$ :

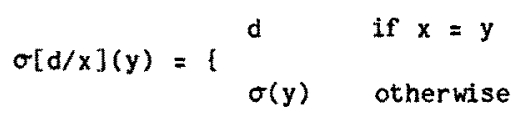

The meaning of an expression is defined by the semantic function

$$
\text { B: 〈exp〉 } \rightarrow \text { AENV } \rightarrow \mathrm{P}(\mathrm{ENV} \rightarrow \mathrm{D})
$$

We write $B_{\sigma}[E]$ for $B(E)(\sigma)$. Note that due to the fact that agent identifiers stand for sets of stream processing functions, this particular semantic model is chosen.

Let $f=\langle a g e n t-i d\rangle$

$$
\begin{aligned}
& B_{\sigma}\left[f\left(E_{1}, \ldots, E_{n}\right)\right]=\text { def } \\
& \text { I } h: \text { ENV } \rightarrow D: \exists g \in \sigma[f], h_{1} \in B_{\sigma}\left[E_{1}\right], \ldots, h_{n} \in B_{\sigma}\left[E_{n}\right]: \\
& \left.\forall e \in E N V: h(e)=B\left(h_{1}(e), \ldots, h_{n}(e)\right)\right\} \\
& B_{\sigma}[\text { rest } E]=\operatorname{def}\left\{h: E N V \rightarrow D: \exists g \in B_{\sigma}[E]: \forall e \in E N V: h(e)=\operatorname{rest}(g(e))\right\} \\
& B_{\sigma}[\text { first } E]=\operatorname{def}\left\{\mathrm{h}: \mathrm{ENV} \rightarrow \mathrm{D}: \exists \& \in \mathrm{B}_{\sigma}[\mathrm{E}]:\right. \\
& \forall e \in E N V: h(e)=\operatorname{first}(g(e)) \\
& B_{\sigma}[\text { isempty } E]=\operatorname{def}\left[\mathrm{h}: \mathrm{ENV} \rightarrow \mathrm{D}: \exists g \in B_{\sigma}[E]:\right. \\
& \forall e=\text { ENV: } h(e)=\text { isempty }(g(e)) \text { ] }
\end{aligned}
$$




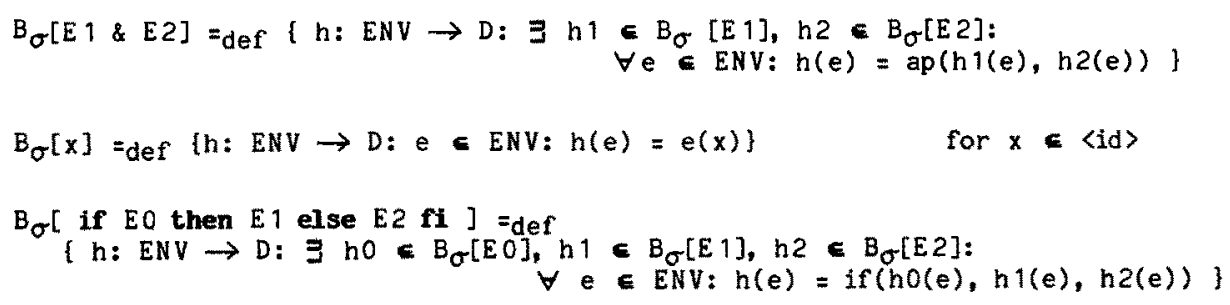

where if $: D^{3} \rightarrow D$ is defined by

$$
\begin{aligned}
& \text { if }(d 0, d 1, d 2)=\left\{\begin{array}{cl}
d 1 & \text { if } d 0=\text { true } \\
d 2 & \text { if } d 0=\text { false } \\
4 & \text { otherwise }
\end{array}\right. \\
& B_{\sigma}\left[\left(E_{1}, \ldots, E_{n}\right)\right]=\operatorname{def}\left(h: E N V \rightarrow D: \exists h_{1} \in B_{\sigma}\left[E_{\eta}\right], \ldots, h_{n} E B_{\sigma}\left[E_{n}\right] ;\right.
\end{aligned}
$$

The meaning of formulas is defined by the semantic function

$$
M:\langle f o r m u l a\rangle \rightarrow A E N V \rightarrow P(E N V \rightarrow \mathbb{B})
$$

We write $M_{\sigma}[H]$ for $M(H)(\sigma)$.

$$
\begin{aligned}
& M_{\sigma}[E 1=E 2]=\operatorname{def}\left(h: E N V \rightarrow \mathbb{B}: \exists g 1 \in B_{\sigma}[E 1], g^{2} \in B_{\sigma}[E 2]:\right. \\
&\forall e \in E N V: h(e)=(g 1(e)=g 2(e))\}
\end{aligned}
$$

For boolean expressions $E$ we write often just $E$ instead of $E=$ true.

$$
\begin{aligned}
M_{\sigma}[H 1 \wedge H 2]=\operatorname{def}\left\{h: E N V \rightarrow \mathbb{B}: \exists h 1 \in M_{\sigma}[H 1], h 2=M_{\sigma}[H 2]:\right. & \forall e \in E N: h(e)=(h 1(e) \wedge h 2(e))\}
\end{aligned}
$$

We often write ", instead of " $\wedge$ ". Analogous definitions are assumed for the remaining logical connectives.

$$
\begin{aligned}
& M_{\sigma}[\forall x: H]=\operatorname{def}\left\{h: E N V \rightarrow \mathbb{B}: \exists \text { ho } \in M_{\sigma}[H] \forall e \in E N V:\right. \\
& h(e)=\forall d \in R: h o(e[d / x])]
\end{aligned}
$$

where, $R$ denotes the subset of $D$ that is indicated by the sort $m$.

$$
M_{\sigma}[\exists \mathrm{m} x: H]=\operatorname{def} M_{\sigma}[\neg \forall \mathrm{m} x: \neg H]
$$

In agent specifications agent identifiers stand for (sets of) stream processing functions. Every time a stream identifier occurs in a specification another instantiation may be taken, i.e. another stream processing function out of the set of stream processing functions can be chosen.

Given a formula $H$ and an agent-environment $\sigma$ and a data environment $e$ we say $H$ is valid for $\sigma$ and $e$ and write 
$(\sigma$, e) $\vDash H$

if

$\forall \sigma_{0} \in$ AENV: $\sigma 0 \leqq \sigma \Rightarrow 3 h \in M_{\sigma 0}[H]: h(e)=$ true

Here $\sigma 0 \leqq \sigma$ is used in the sense of pointwise subset relation.

The choice of this particular definition of validity is essentially motivated by concepts of implementation and program design as explained in the following sections: An agent may be used in networks of communicating agents and may occur several times. For each occurrence one arbitrary function out of the set of allowed functions may be chosen. However, the formula even holds if one uses an implementation of the resp. agent (1.e. a subset from the set of possible agents).

If $H$ does not contain free data identifiers, the validity is independent of $e$ and we write

$\sigma: H$

In the following we use $\bar{x}$ (and $\bar{y}$ resp.) as abbreviation for tuples of declarations, i.e. for phrases of the syntactic unit <dec-tuple>. Let $\bar{x}$ stand for

$s_{1} x_{1}, \ldots, s_{n} x_{n}$

where the $s_{i}$ specify the sort of the identifiers $x_{i}$. Let furthermore $x$ (and $y$ resp.) stand for $\left(x_{1}, \ldots, x_{n}\right)$. Now it is defined, under which circumstances an agent specification is fulfilled by an environment. Given an agent specification

agent $f=$ input $\bar{x}$, output $\bar{y}, \quad H$ end

where we assume that in $H$ only agent identifiers and the identifiers from $\bar{x}$ and $\bar{y}$ occur, we say for a given agent environment $\sigma$ " $\sigma$ fulfils the specification for $f^{\prime \prime}$ if

$$
\sigma: \forall \bar{x}: \exists \bar{y}: y=f(x) \wedge H
$$

Given a family DEF of definitions of the agent, $f_{1}, \ldots, f_{k}$

agent $f_{1}=\ldots H_{1}$ end $\ldots$ agent $f_{k}=\ldots H_{n}$ end

an agent environment

$$
\sigma:\left\{f_{1}, \ldots, f_{k}\right\} \rightarrow P(\text { AGENT })
$$

is called consistent and we write $\sigma \neq D E F$ if $\sigma$ fulfils all the agent specifications for $f_{1}, \ldots, f_{k}$. The semantics of the family of agent definitions then is given by the sets of all agent environments that fulfil the specification, i.e. by

$$
\left\{\sigma:\left\{f_{1}, \ldots, f_{k}\right\} \rightarrow P(A G E N T): \sigma \neq D E F\right\}
$$

This way every family of agent specifications $f_{i}$ is semantically defining a set of environments, $i$. e. a set of families (mappings from identifiers to sets) of sets of stream-processing functions. 
In the context of equational ("algebraic") specifications of families of agents similar questions arise as for the algebraic specification of abstract (data) types in hierarchies. One may introduce notions like persistency, hierarchy completeness (sufficient completeness), or hierarchy consistency in (algebraic) specifications of agents.

\subsection{Teaporal Logics}

A highly developed notation and calculus for reasoning on (sets of) sequences is (linear time) temporal logic. Temporal logic mainly has been advocated and used for reasoning about complete concurrent systems cooperating via shared memory. Since agents are functions on sequences temporal logic should provide a framework also for agent specifications. Since one has to reason in agent specifications about several sequences in one formula, the temporal logic framework has to be slightly generalized. Three temporal operators are introduced.

Let $s$ be a stream identifier and $H$ be a predicate where $s$ is used as a stream. Then we define

$$
\begin{aligned}
& \circ s_{1}, \ldots, s_{k}: H={ }_{\operatorname{def}} H\left[\left(\text { rest } s_{1}\right) / s_{1}, \ldots,\left(\text { rest } s_{k}\right) / s_{k}\right] \\
& 0 s_{1}, \ldots, s_{k}: H=\operatorname{def} \quad \forall i \in \mathbb{N}: H\left[\left(\text { rest }^{i} s_{1}\right) / s_{1}, \ldots,\left(\text { rest }^{i} s_{k}\right) / s_{k}\right] \\
& \diamond s_{1}, \ldots, s_{k}: H={ }_{\operatorname{def}} \quad \exists i \in \mathbb{N}: H\left[\left(\text { rest }^{i} s_{1}\right) / s_{1}, \ldots,\left(\text { rest }^{i} s_{k}\right) / s_{k}\right]
\end{aligned}
$$

Here rest is $^{i}$ assumed to by defined by

$$
\operatorname{rest}^{0} s=s, \quad \operatorname{rest}^{i+1} s=\operatorname{rest}\left(\operatorname{rest}^{i} s\right)
$$

By $\mathrm{H}[\mathrm{E} / \mathrm{S}]$ we denote the expression that is obtained by replacing all occurrences of $s$ in $H$ by the expression $E$.

In terms of our semantic definitions the meaning of the temporal operators is given by:

$$
\begin{aligned}
& M_{\sigma}\left[\circ s_{1}, \ldots, s_{k}: H\right]=\operatorname{def}\left\{h: E N V \rightarrow \mathbb{B}: \exists \text { ho } \subseteq M_{\sigma}[H] \forall e \in\right. \text { ENV: } \\
& \left.h(e)=\operatorname{ho}\left(e\left[r e s t\left(s_{1}\right) / s_{1}, \ldots, \operatorname{rest}\left(s_{k}\right) / s_{k}\right]\right)\right\} \\
& M_{\sigma}\left[\left[s_{1}, \ldots, s_{k}: H\right]=\operatorname{def}\left\{h: E N V \rightarrow \mathbb{B}: \exists h_{0} \equiv M_{\sigma}[H] \forall e \equiv E N V:\right.\right. \\
& \left.h(e)=\forall 1 \in \mathbb{N}: h_{0}\left(e\left[\operatorname{rest}^{i}\left(s_{1}\right) / s_{1}, \ldots, \operatorname{rest}^{i}\left(s_{k}\right) / s_{k}\right]\right)\right] \\
& M_{\sigma}\left[\circ s_{1}, \ldots, s_{k}: H\right]=\operatorname{def}\left\{h: E N V \rightarrow \mathbb{B}: \exists \text { ho } \in M_{\sigma}[H] \forall\right. \text { e ENV: } \\
& \left.\left.h(e)=\exists i \in \mathbb{N}: h 0\left(\operatorname{erest}^{i}\left(s_{1}\right) / s_{1}, \ldots, \operatorname{rest}^{i}\left(s_{k}\right) / s_{k}\right]\right)\right]
\end{aligned}
$$

With these temporal operators one can give specffications without any use of "recursion". For instance the example onestream then reads

agent onestream $=$ output strean nat $r, \quad[r$ : first $r=1$ end

Temporal formulas therefore stand for infinite formulas and thus can be seen to allow often a more explicit way of specifications. For some of the examples of section 2 one can obtain more explicit specifications by temporal logics: 
agent infinite $=$ output stream bool $r:([] r$ first $r) \vee([] r: \neg$ first $r$ ) end

agent addstream = input stream nat $a$, stream nat $b$, output stream nat $r$ :

$[a, b, r$ : first $r=$ first $a+$ first $b$ end

agent any $=$ output stream $r, \quad \square r$ : (first $r \vee \neg$ first $r$ ) end

For other examples such as the agent merge or the agent schedule, more explicit (nonalgebraic) specifications using temporal logic are less obvious. An appropriate, more powerful temporal logic for specifying communicating agents without algebraic (recursive) equations seems one of the interesting questions to be looked at.

Possible candidates for such an extended temporal logic are for instance versions of the until operator, the (iterated) combine operator, and the fixed point operator for logical formulas as suggested in [Barringer et al. 85]. However, it seems not clear so far, whether an algebraic style of specifications or such a more logic-oriented style of specifications is more appropriate. For the moment I prefer a formalism which supports both styles.

\section{Agent Specifications Describing Algorithms}

The formalism introduced so far was explained as a specification tool. Nevertheless agent specifications of particular syntactic forms can be seen as programs. Such specifications are called algorithmic agents.

\subsection{Algorithmic Agents}

An algorithmic agent is an agent specification of the following syntactic form:

$$
\text { agent } f=\text { input } \bar{x} \text {, output } \bar{y}, \exists \bar{z}: H_{1} \wedge \ldots \wedge H_{j} \text { end }
$$

The set $x$ of identifiers of $\bar{x}$ are called input ports, the set $y$ of identifiers in $\bar{y}$ are called the output ports, the set $\bar{z}$ of identifiers in $\bar{z}$ are called the internal ports.

The formulas $H_{j}, 1 \leq i \leq j$ are assumed to be of the form

$$
\left(a_{i}=T(\stackrel{i}{i}) \vee \ldots \vee a_{i}=T \underset{p}{(i)}\right)
$$

where $a_{i}$ is called the left-hand side of the clause $H_{i}$ and the $a_{j}$ are tuples of

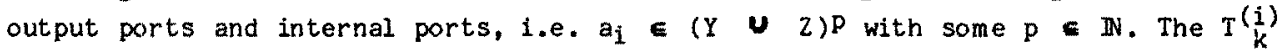
are assumed to be expressions. Every identifier in $Y U Z$ must occur in exactiy one left-hand side $a_{i}$. A system of agents is called algorithmic, if all agents of the system are algorithmic.

Algorithmic agent specifications can be trivially translated into applicative multiprograms written in programming languages such as AMPL (cf. [Broy 81]) for which an operational (reduction) semantics is available. This way algorithmic specifications define algorithms. In a system of agent specifications it is said that "the agent $f$ uses the agent $g$ " if 
(1) $g$ occurs in the body of the specification of agent $f$, or

(2) $g$ is used by an agent $h$ that is used by $f$.

If an agent $f$ uses itself, then it is called recursive agent specification. If an algorithmic agent specification contains equations with stream identifiers occurring on both the left-handside and the right-handside of equations between streams then we speak of recursive (algebraic) equations for streams.

Every algorithmic agent defines a network (a data flow graph), if we take $X U Y$ $U Z$ as arcs and introduce a node for every clause $H_{i}$. The arcs have as sources the nodes of the clauses where they appear on the lefthand side. If the agent specification is recursive, then the defined network is infinite, if the agent specification is not recursive but contains recursive stream equations, then the network is cyclic. If the algorithmic specification is neither recursive nor is containing recursive stream equations, then the network is a finite, acyclic (directed) graph. Examples are given below.

\section{Design Issues}

After having introduced a particular language for the specification of agents, in this section a number of design issues are treated that give some insights, how the language for specifying agents can be used.

\section{1. A Combinatorial/Functional Notation for Agents}

So far the specifications are written using (internal) identifiers for data objects. In this section a functional style notation is introduced that allows to combine given agent specifications by sequential composition, parallel composition and feedback.

In the following we assume the two agent specifications to be given:

agent $a_{1}=$ input $\bar{x}$, output $\bar{y}, H 1$ end

agent $a_{2}=$ input $\overline{x^{2}}$, output $\overline{y^{2}}, H_{2}$ end

where $a 1$ has $n 1$ input lines and $m 1$ output lines and a2 has $n 2$ input lines and $m 2$ output lines. We assume, that $\overline{x 1}, \overline{x_{2}}, \overline{y 1}, \overline{y 2}$ are pairwise disjoint w.r.t. the (sets of) identifiers they contain.

The parallel composition of the two agents a1 and a2 is written by

a) || a2

where $a_{3}=a_{1} \|$ a2 has $n 1+n 2$ input lines and $m 1+m 2$ output lines and it is specified by 
agent $a 3=$ input $\overline{x_{1}}, \overline{x_{2}}$, output $\overline{y_{1}}, \overline{y_{2}}, \mathrm{H}_{1} \wedge \mathrm{H}_{2}$ end

For simplicity let us assume that now $m 1=n 2$. The sequential composition of the two agents $a 1$ and $a 2$ is written by

$$
\text { a } 1 \cdot \mathrm{a} 2
$$

where $a_{4}=a 1 . a 2$ is an agent specification with $\mathrm{n} 1$ input lines and $\mathrm{m} 2$ output lines. It is defined by

$$
\text { agent } a_{4}=\text { input } \bar{x} 1, \text { output } \overline{y^{2}}, \exists \overline{x_{2}}, \overline{y_{1}:} \times 2=y_{1} \wedge H_{1} \wedge H_{2} \text { end }
$$

The feedback of an agent is defined by

$$
c_{j}^{i} a
$$

where the agent $a$ is assumed to be given by the specification (with $1 \leq i \leq n$, $1 \leq j \leq m$ )

agent $a=$ input $s_{1} x_{1}, \ldots, s_{n} x_{n}$, output $r_{1} y_{1}, \ldots, r_{m} y_{m}, H$ end and the agent a5 $=c_{j}^{i}$ a has $n-1$ input lines and $m$ output lines. It is defined by the agent specification

$$
\begin{aligned}
& \text { agent } a 5=\text { input } s_{1} x_{1}, \ldots, s_{i-1} x_{i-1}, s_{i+1} x_{i+1}, \ldots, s_{n} x_{n} \text {, } \\
& \text { output } r_{1} y_{1}, \ldots, r_{m} y_{m}, \quad \exists s_{i} x_{i}: x_{i}=y_{j} \wedge H \\
& \text { end }
\end{aligned}
$$

Note that the composition of algorithmic agents always leads to algorithmic agents again.

The agent store may be turned into an algorithmic agent (assuming algorithmic agents for switch and schedule) by:

agent store = input stream data $d$, stream bool $b$, output stream data $r$,

$\exists$ stream data $s, z:(z, r)=\operatorname{switch}(s, b), s=\operatorname{schedule}(d, r$, true \& $b)$ end

This agent store may be represented by the data flow diagram

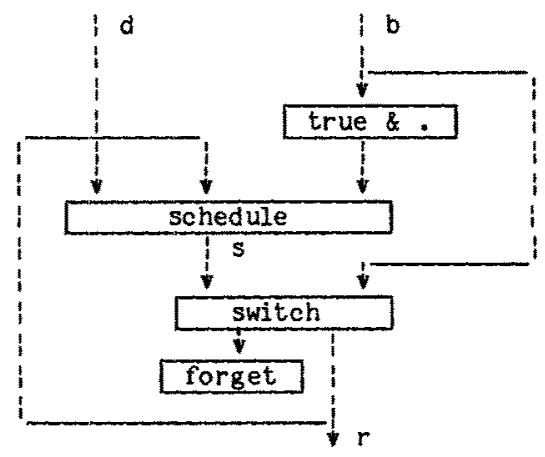


It can be also expressed by

$$
c_{1}^{2}((I \text { i| } I \text { i a) })(\text { schedule } \| I) \cdot \operatorname{switch} \cdot(\text { forget } \| I))
$$

where $I$ is the identity function and the agents forget and $a$ are the trivial agents specified by

agent forget $=$ input strean data $d$, true end

agent $a=$ input stream bool b, output strean bool b1, b2,

$$
b 1=\text { false \& } b, \quad b 2=b \quad \text { end }
$$

\subsection{Correctness of Agents}

During a design process of a distributed system a sequence or even a tree of agent specifications are produced. The final agent specification should be a program, i.e. an algorithmic agent, that must be correct w.r.t. the initial agent specification. In the most restrictive approach one could define correctness in the following way: An agent specification A1 is totally correct w.r.t. an agent specification $A O$, iff the set of functions denoted by A1 is identical to the set of functions denoted by AO. However, obviousiy this approach is too restrictive. More liberal notions of correctness have to be used and can be used much more flexible in the design process.

\subsubsection{Partial Correctness}

Partial correctness of concurrent, communicating systems is a so-called safety property: a program is partially correct (w.r.t. to some requirement specification) iff it never produces wrong results. This does not exclude that it diverges always immediately and therefore does not produce any results. Given an agent specification $A 1$, that defines a set $F 1$ of stream processing functions, and a requirement specification $A O$, that defines a set $F O$ of stream processing functions, $A 1$ is called partially correct w.r.t. A0, iff

$$
\forall f 1 \subset F 1 \exists \text { fo }<F O: f 1 \leq f 0
$$

This formula defines a preordering on sets that is also used in powerdomains. It is abbreviated by F1 [E FO. Accordingly for a partially correct agent every output stream is a prefix of an output stream included in the requirement specification.

\subsubsection{Robust Correctness}

Robust correctness is a typical liveness property: if a certain amount of output is guaranteed by the specification, then at least the same amount of information is to be guaranteed by a robust correct program. Given an agent specification A1 defining a set $F 1$ of stream processing functions and an agent specification $A 0$ defining a set FO of stream processing functions, then $A 1$ is called robustly correct w.r.t. A0, iff

$$
\forall \mathrm{fl}_{1} \in \mathrm{F}_{1} \equiv \mathrm{fO} \in \mathrm{FO}: \mathrm{fO}[\mathrm{f} 1
$$

This is a preordering on sets that is also used in powerdomains. It is abbreviated by FO [M F1. Accordingly for a robustly correct agent every output contains a prefix of some output included in the requirement specification. If according to the specification there may be some divergence and no moreoutput, then a robust correct implementation may include some error message at that point. 


\subsubsection{Correct Implementations}

During the design process of a program for a given specification Ao typically particular design decisions are taken: specific algorithmic solutions are envisaged and certain nondeterministic alternatives are excluded. Accordingly for an agent specification $A 1$ defining the set of functions $F 1$ and the agent specification $A O$ defining the set of agents $F O$ the agent $A 1$ is called a (correct) implementation of $A O$ if

$$
\text { F1 } 1 \text { FO }
$$

This definition reflects a very essential point of view of nondeterministic concurrent programs: a correct implementation may not include all nondeterministic possibilities but restrict itself to certain subsets, i.e. by choosing a particular scheduling strategy. Immediately one obtains the following lemma:

Lemma: If $A 1$ is a correct implementation of $A 0$, then

- A1 is partially correct w.r.t. AO

- A1 is robustly correct w.r.t. A0

Note that the converse statement does not hold: robust and partial correctness do not imply the correctness of an implementation.

\subsection{Recursively Defined Algorithmic Agents}

For recursively defined algorithmic agent specifications the semantic definitions are very liberal: the semantics is not restricted to least (defined) fixed points but considers the class of all fixed points. Trivially if one restricts the meaning of a recursively defined algorithmic agent by transition to a least (defined) fixed point semantics, then this represents a correct implementation of the given agent.

An algorithmic agent may trivially be translated into an applicative language for multiprocessing like AMPL (cf. [Broy 83b]). Then, however, a particular fixed point theory is used, while in the specification all fixed points are included. This restriction can be also expressed in our specification language by prefixing algorithmic specifications by rec. The system of agents

rec agent $f_{1}=\ldots E_{q}$ end $\ldots$ rec agent $f_{n}=\ldots H_{n}$ end

has the meaning $\sigma_{\mathrm{rec}}$, where

$$
\sigma_{\text {rec }} \approx\left\{\sigma:\left\{f_{1}, \ldots, f_{n}\right\} \rightarrow \text { P(AGENT) }\right\}
$$

is the $\leq$-least ( $\leq$ taken pointwise) function which is a LM-least and [EM-least fixed point of the equations $E_{i}$ with

$$
\sigma_{\text {rec }} \| \forall \bar{x}_{1} \exists \bar{y}: y=f_{i}(x) \wedge H_{i}
$$

for $1 \leq i \leq n$. Here $L_{M}$ is the preordering defined in the previous section. The preordering LEM is defined by LE $\cap$ LM. 
The mathematical soundness and consistency of this definitions is implied by [Broy $84 \mathrm{c}]$.

Trivially, if one prefixes a given family DEF of agent specifications by rec, then one obtains a correct implementation of DEF: the least fixed points in the sense above provide a subset of the set of fixed points. Therefore the final step of a program development, viewing an algorithmic specification as a program, is trivially correct.

\subsection{Transformations of Agent Specifications}

The mathematical semantics of agent specifications and the definition of correct implementations clearly define what may be called a (partially, robustly) correct transformation step for a specification: the transformed specification must be a (partially, robustly) correct implementation of the initial program. On this basis a calculus for transformation rules can be developed. It comprises essentially the rules of inference of predicate logic, the algebraic axioms of streams and the basic rules of the used programming constructs.

Note, however, that due to the existence of nondeterministic agent specifications all laws of predicate logic where textual copying is involved have to be reconsidered and if necessary modified. Applying rules of inference where agent identifiers are copied may lead to incorrect implementations. Consider for instance the formula

$$
y=f(x) \wedge z=y
$$

which is essentially different from

$$
y=f(x) \wedge z=f(x)
$$

if $f$ is a nondeterministic agent.

Now a simple example for the transformation of an agent specification is given. It essentially shows a development starting with recursive agents towards recursive stream equations.

Example: From recursion on functions to recursion on streams.

The agent sumso computes the partial sums of a stream:

agent sums $O=$ input stream nat $s$, output stream nat $r, r=h e l p(s, 0)$ end

agent help $=$ input stream nat $s$, nat $n$, output stream nat $r$, $r=n$ \& help (rest $s, n+$ first $s$ ) end

The agent sumso or more precisely the agent help is defined recursively. It can be graphically represented by the diagram

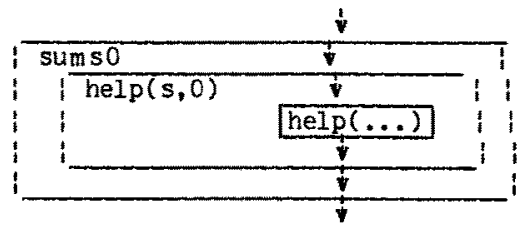


The agent sumso is extensionally equivalent to the agent sums 1:

agent sum 1 = input stream nat $s$, output stream nat $r, r=0$ \&addstream $(r, s)$ end

The agent sums 1 is defined by recursion on streams. It can be seen as defining a data flow diagram:

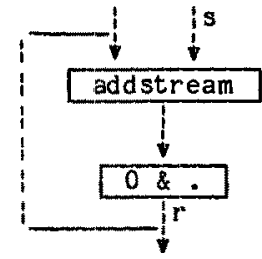

The agents sums0 and sums 1 may also be represented by the term

$$
c_{1}^{1} \text { (addstream - zero) }
$$

where zero is defined by

agent zero $=$ input stream nat $s$, output stream nat $r, r=0 \& s$ end

$$
\text { end of example }
$$

The example may be generalized to the following development rule: If an agent contains an equation with streams $r$, $s$ of the form (with some given agent $f$ ):

(*) $r=f(s)$

and one can prove the formulas (with some given agent g):

(1) $f(s)=g(f(s), s)$

(2) $\forall s: r^{1}=g\left(r_{1}, s\right) \wedge r^{2}=g\left(r^{2}, s\right) \Rightarrow r 1=r^{2}$

then (*) can be replaced by the equation

$$
r=g(r)
$$

The condition ( 1 ) states the requirement that $f(s)$ is a fixed point of $g$, the requirement (2) in addition requires that $g$ has an unique fixed point.

\section{Example: (Continued)}

For verifying the applicability conditions (1) and (2) in our example above, one has to prove 
(1) $\operatorname{help}(s, n)=n$ addstream $(h e l p(s, n), s)$

The condition (1) can be proved by structural induction on s:

(i) if $s=\langle\boldsymbol{t}\rangle$, then (and similarly for $s=$ E):

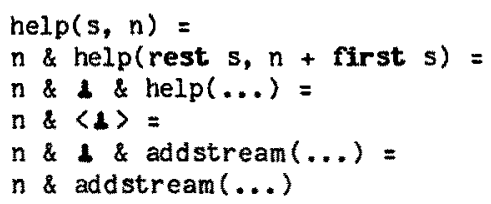

(ii) if $s=x \&$ sn (where $x \in \mathbb{N}$ ) and (1) holds for $\mathrm{sn}$ :



For infinite $s$ the continuity of the involved functions proves the result. Finally one has to prove that the equation

$$
r=n \& \operatorname{addstream}(r, s)
$$

has an unique fixed point for any $s$. Again this can be proved by induction on the length of $s$ :

(i) if $s=\langle\mathbf{s}$ then the equation implies

$$
\text { addstream }(r,\langle\boldsymbol{k}\rangle)=\langle\boldsymbol{k}\rangle \text {, and hence } r=n \&\langle\boldsymbol{k}\rangle
$$

(ii) if for $s$ the solution of the equation above is unique, then with $x \in \mathbb{N}$

$$
r=n \& \text { addstream }(r, x \& s)
$$

one gets

$$
r=n \text { (first } r+x) \& \text { addstream (rest } r, s \text { ) }
$$

which now can be replaced by the equations

$$
r=n \& t, \quad t=(n+x) \& \text { addstream }(t, s)
$$

According to our induction hypothesis $t$ is uniquely determinated for finite streams s. For infinite streams the uniqueness of the fixed point of the equation follows from a continuity argument: the fixed point depends continuously on $s$.

\section{end of example}

of course, the transformation rule above is only one simple example for transformations of agent specifications. It can be seen immediately that there is a rich class of transformation rules for agent specifications. Many of the transformation rules for sequential programs ( $c f$. [Broy 84d]) can be adapted also for agent specifications. 


\subsection{Verification of Agent Specifications}

Proving for two given agents AO, A1 that A1 is a (partially, robustly) correct implementation of AO represents the classical case of verification. After resp. renaming of the input and output identifiers of $A 1$, one basically has to show that the specifying predicate of A1 implies the specifying predicate of A0. Formally this again can be done by transforming AO to A1 or more precisely deducing AO from A1. Moreover one may also think of particular calculi for the verification of agents. For instance calculi dealing just with particular aspects of communicating agents may be developed such as calculi for partial or robust correctness.

\subsection{Structured Programming with Agent Specifications}

Trivially many different specifications can be given for one set of stream processing functions. Apart from different ways of writing equivalent predicates and renaming of local identifiers one could use different ways of structuring a specification.

A complete system of agent specifications consists of a family of agent specifications where for all occurring agent identifiers agent specifications are provided. The relation "agent $a_{i}$ is used in agent $a_{j}$ " then defines a quasiordering, and therefore induces a partial ordering on a quotient structure on the set of agents. This quasiordering is called the macro structure of the system.

Every agent itself contains some structure: the way how the specifying predicate is written. Especially for algorithmic agents this form of the predicate, the number of local identifiers is interesting, because it defines a finite network of communicating agents. This structure is called the micro structure of (the components of) the system.

Clearly the micro structure of an agent may again be seen as the macro structure of a (sub-)system with some further micro structure. Due to the use of recursive agents, such a transition from macro to micro views may be done up to infinity.

An important transformation rule that connects the micro structure with the macro structure is the unfold/fold rule for agents: Given an agent

agent $a O=$ input $\bar{x} \overline{0}$, output $\bar{y} \overline{0}$, EO end

and an occurrence of the agent a0 in an equation

$$
t 1=a 0(t 2)
$$

then one may "unfold" the agent specifications, i.e. one may replace the equation (*) by the formula

(**) $\exists \bar{x} \overline{0}, \overline{y 0}: \quad t 1=y 0 \wedge x 0=t 2 \wedge E 0$

of course it is assumed that name clashes do not appear.

Even the reverse of the rule above is possible: the formula (**) may be replaced by the equation (*), provided $(* *)$ is occurring outside of the body of the agent a0. If ao may be graphically represented by a finite data flow network 


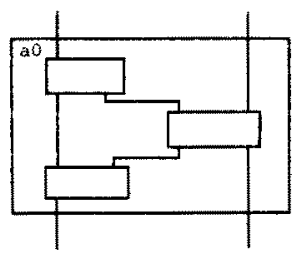

and an agent a uses a0;

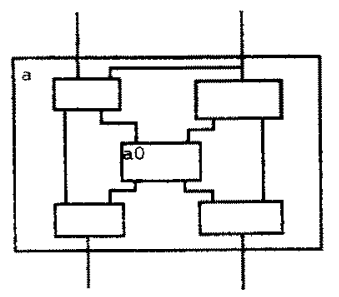

then by the rule one obtains for the agent a:



This way algorithmic agents are transformed into again algorithmic agents.

\section{Application to Other Models for Concurrency}

Specifications of concurrent communicating agents are based on the model of stream processing functions. This is essentially a model with implicit buffering message passing. Other important models are handshaking message passing or shared memory systems. An immediate question is, whether the specification method for agents can be applied for the other models, too.

The answer is rather simple: there exist semantic models both for shared memory as well as for handshake communication in terms of stream-processing functions (cf. [Broy 83, 84a, 84b]). Therefore one may simply write agent specifications, that specify stream-processing functions that represent the semantics of programs written in those programming languages according to those semantic models.

Example: Specification of producer and consumer

According to the semantic model for shared memory as given in [Broy 84a] components of a system of parallel programs working on some shared memory the access to which is protected by conditional critical regions may semantically be 
modelled by (sets of) functions mapping streams of states to streams of states and "rejects". For instance the specification of the producer/consumer problem may read as follows (here $x, y, z, q$ denote programming varlables):

agent producer $=$ input stream state $s$, output stream (state $\boldsymbol{U}$ \{REJECT\}) $r$,

first $r=$ (first $s)\left[x_{0} / x\right] \wedge$

[] $r, s: \circ r, s$ : first $r=$ (first $s)[$ next $(($ first $s)(x)) / x$, $\operatorname{app}(($ first $s)(q),($ first $s)(x)) / q]$ end

agent consumer $=$ input stream state $s$, output stream (state $u$ \{REJECT\}) $r$,

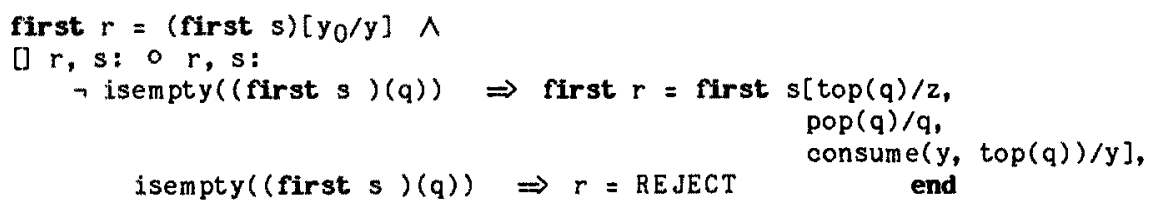

Here the notation $s[d / x]$ is used as the notation for updating the state $s$ by replacing the value for the identifier $x$ by $d$.

Procedural programs that fulfil this specification then read:

producer:

$\mathrm{x}:=\mathrm{x}_{0}$;

while true do await true then $q:=\operatorname{app}(q, x)$ endwait;

$x:=\operatorname{next}(x)$

od

consumer:

$\mathrm{y}:=\mathrm{y}_{0}$;

while true do await $\rightarrow$ isempty $(q)$ then $q, z:=p \circ p(q)$, top $(q)$ endwait;

$y:=$ consume $(y, z)$

ad

Here the specification does not look very convincing: It is textually larger and (since less familiar) seems harder to understand than the programs. However a tuned notation for the specification might change this view. Writing $x$ " for the input value of a program variable (i.e. for (first $s)(x)$ ) and $x^{\prime}$ for its output value (i.e. for (first $r)(x)$ ) and dropping $r, s$ from temporal formulas one obtains: sm-agents (shared memory agents). It is just consequent to add the information which variables are assumed to be shared:

sm-agent producer $\equiv$ shared $q$, $x^{\prime}=x_{0} \wedge\left([] \quad 0 \quad\left(x^{\prime}=\operatorname{next}\left(x^{\prime}\right) \wedge q^{\prime}=\operatorname{app}\left(q^{\prime}, x^{\prime}\right)\right) \quad\right.$ end

sm-agent consumer $\equiv$ shared $q$,

$$
\begin{aligned}
y^{\prime}=y_{0} \wedge \quad \square \quad\left(\left(-1 \operatorname{sempty}\left(q^{\prime}\right) \Rightarrow\right.\right. & \left(z^{\prime}=\operatorname{top}\left(q^{\prime}\right) \wedge\right. \\
q^{\prime}=\operatorname{pop}\left(q^{\prime}\right) \wedge & \wedge \\
\left.y^{\prime}=\text { consume }\left(y^{\prime}, z^{\prime}\right)\right) \wedge & \text { end }
\end{aligned}
$$

end of example

In a similar way one may develop tuned notations for CSP-like programs or CCS-like programs by using the stream-based semantic models for CSP/CCS as given in [Broy $83 a, 84 a$. 


\section{Concluding Renarks}

The approach of agent specifications reflects an attempt to bring together distinct research directions: algebraic (equational) specifications, denotational models for concurrent, communicating systems, temporal logic, functional programming and program development by transformations.

The presented approach to the specification of concurrent communicating systems includes a number of design decisions that should be shortly recapitulated in the end and some justification should be given, too.

The chosen semantic model are sets of continuous functions mapping tuples of streams to tuples of streams. Why tuples of streams are considered is obvious. Considering sets of functions instead of relations or set-valued functions might be less clear (for a more detailed justification see for instance [Broy 81]). It is done for avoiding some subtle problems in connection with definitions of streams by fixed points over nondeterministic functionals. Whether the restriction to continuous functions is actually always appropriate, however, seems less clear.

The chosen logical framework is basically algebraic: All properties are specified by equations. The inclusion of temporal logic can just be seen as a notational variant being appropriate since sequence-like structures like streams are used. May be it is important to point out that the logic is two-valued in spite of the existence of partial functions (in the disguise of total functions with \& as result) and of nondeterministic agents.

The chosen concept of validity of specifications represents a very subtle point. The validity of formulas containing nondeterministic terms can be defined in several ways (by several modalities). Our choice represents a compromise between universal validity (a formula has to be valid for all nondetermistic alternatives) and existential validity (a formula has to be valid for only one nondeterministic alternative). It is justified by the interpretation of formulas as specifying networks of nondeterministic agents.

May be that the most important properties of a specification method are not only the underlying theoretical concepts but more pragmatic issues such as readability, tractability, support for structuring, possibilities of visual aids and machine support. In this light the presented approach seems rather attractive. The essential principle that every family of agents can be also considered (and formally speciffed) as a network and vice versa, the integration of pure specification constructs and algorithmic views into one framework let expect that the given approach can be further developed into a flexible and practically helpful tool.

What has been presented in the previous section rather can be seen as a first attempt to develop a modular specification and design method for communicating concurrent systems than a fully worked out methodology. Much remains to be done until such an approach actually will work practically. 


\section{References}

[Barringer et al. 85]

H. Barringer, R. Kuiper, A, Pnueli: A Compositional Temporal Approach to a CSP-like Language. (Unpublished manuscript)

\section{[Broy 81$]$}

M. Broy: A Fixed Polnt Approach to Applicative Multiprogramming. In: M. Broy, G. Schmidt (eds.): Theoretical Foundations of Programming Methodology, Reidel Publ. Comp. $1982,565-623$

\section{[Broy 83a]}

M. Broy: Denotational Semantics of Communicating Processes based on a Language for Applicative Multiprogramming. IPL 17:1 1983, 29-38

\section{[Broy 83b]}

M. Broy: Fixed Point Theory for Communication and Concurrency. In: D. Bjorner (ed.): IF IP TC 2 Working Conference on Formal Description of Programming Concepts II, Garmisch, June 1982, Amsterdam-New York-Oxford: North Holland Publ. Company $1983,125-147$

[Broy 83c]

M. Broy: Applicative Real Time Programming. In: R.E.A. Mason (ed.): Information Processing 83, 259-264

[Broy 84a]

M. Broy: Semantics of Communicating Processes. Information and Control (to appear)

\section{[Broy 84b]}

M. Broy: Denotational Semantics of Concurrent Programs with Shared Memory. In: M. Fontet, K. Mehihorn (eds.): STACS 84, Lecture Notes in Computer Science 166, Berlin-Heidelberg-New York: Springer 1984, 163-173

[Broy 84c]

M. Broy: On the Herbrand Kleene Universe of Nondeterministic Computations. In: M.P. Chytil, V. Koubeck (eds.): Mathematical Foundations of Computer Science 1984. Lecture Notes in Computer Science 176, Berlin-Heidelberg-New York-Tokyo: Springer 1984, 214-222

\section{[Broy $84 d$ ]}

M. Broy: Algebraic Methods for Program Construction: The Project CIP. In: P. Pepper (ed): Program Transformation and Programming Environments. NATO ASI Series. Series F: 8. Berlin-Heidelberg-New York-Tokyo: Springer 1984, 199-222

[Broy, Wirsing 82]

M. Broy, M. Wirsing: Partial Abstract Types. Acta Informatica 18, 1982, 47-64

\section{[Dennis 74]}

J.B. Dennis: First Version of a Data Flow Procedure Language. In B. Robinet (ed.): Colloque sur la Progammation, Lecture Notes in Computer Science 19, Berlin-Heidelberg-New York: Springer 1974, 362-367

[Hehner 84]

E.C.R. Hehner: Predicative Programming Part I+II. CACM 27:2 (1984) 134-151 
[Hoare et al. 81]

C.A.R. Hoare, S.D. Brookes, A.W. Roscoe: A Theory of Communicating Sequential Processes, Oxford University Computing Laboratory, Programming Research Group, Technical Monograph PRG-21, Oxford 1981

[Kahn, MacQueen 77]

G. Kahn, D. MacQueen 77: Coroutines and Networks of Parallel Processes. In: Proc. of the IF IP Congress 77, Amsterdam:North-Holland 1977, 994-998

[Keller 78]

R. M. Keller: Denotational Models for Parallel Programs with Indeterminate Operators. In: E. J. Neuhold (ed.): Formal Description of Programming Concepts. Am sterdam: North-Holland 1978, 337-366

[Milner 80a]

R. Milner: A Calculus of Communicating Systems. Lecture Notes in Computer Science 92, Berlin-Heidelberg-New York: Springer, 1980

[Plotkin 76]

G. Plotkin: A Powerdomain Construction. SIAM J. Computing 5, 1976, 452-486

[Pnueli 77]

A. Pnueli: The Temporal Logic of Programs. Proc 18th FOCS, Providence, 1977, 46-57

[Pratt 78 ]

V.R. Pratt: Process Logic. 6th Popl, 1979, 83-100

[Smyth 78]

M. Smyth: Power Domains. J. CSS 16, 1978, 23-36

[Winskel 82]

G. Winskel: Event Structure Semantics of CCS and Related Languages. In: M. Nielsen, E. M. Schmidt (eds.): ICALP 82 Lecture Notes in Computer Science 140, Berlin-Heidelberg-New York 1982, 561-576 